\title{
Distributed State Estimation in Sensor Networks with Randomly Occurring Nonlinearities Subject to Time-Delays
}

\author{
JINLING LIANG \\ Southeast University, China \\ ZIDONG WANG \\ Brunel University, UK \\ BO SHEN \\ Donghua University, China \\ and \\ XIAOHUI LIU \\ Brunel University, UK
}

\begin{abstract}
This paper is concerned with a new distributed state estimation problem for a class of dynamical systems in sensor networks. The target plant is described by a set of differential equations disturbed by a Brownian motion and randomly occurring nonlinearities (RONs) subject to timedelays. The RONs are investigated here to reflect network-induced randomly occurring regulation of the delayed states on the current ones. Through available measurement output transmitted from the sensors, a distributed state estimator is designed to estimate the states of the target system, where each sensor can communicate with the neighboring sensors according to the given topology by means of a directed graph. The state estimation is carried out in a distributed way and is therefore applicable to online application. By resorting to the Lyapunov functional combined with stochastic analysis techniques, several delay-dependent criteria are established that not only ensure the estimation error to be globally asymptotically stable in the mean square, but also guarantee the existence of the desired estimator gains that can then be explicitly expressed when certain matrix inequalities are solved. A numerical example is given to verify the designed distributed state estimators.
\end{abstract}

Authors' address: J. Liang, Department of Mathematics, Southeast University, Nanjing 210096, China. Email: jinlliang@gmail.com

Z. Wang, Department of Information Systems and Computing, Brunel University, Uxbridge, Middlesex, UB8 3PH, United Kingdom. Email: Zidong. Wang@brunel.ac.uk

B. Shen, School of Information Science and Technology, Donghua University, Shanghai 200051, China. Email: shenbodh@gmail.com

X. Liu, Department of Information Systems and Computing, Brunel University, Uxbridge, Middlesex, UB8 3PH, United Kingdom. Email: Xiaohui.Liu@brunel.ac.uk

This work was supported in part by the National Natural Science Foundation of China under Grants 61028008, 60804028 and 61174136, the Qing Lan Project of Jiangsu Province of China, the Project sponsored by SRF for ROCS of SEM of China, the Engineering and Physical Sciences Research Council (EPSRC) of the UK under Grant GR/S27658/01, the Royal Society of the UK, and the Alexander von Humboldt Foundation of Germany.

Permission to make digital/hard copy of all or part of this material without fee for personal or classroom use provided that the copies are not made or distributed for profit or commercial advantage, the ACM copyright/server notice, the title of the publication, and its date appear, and notice is given that copying is by permission of the ACM, Inc. To copy otherwise, to republish, to post on servers, or to redistribute to lists requires prior specific permission and/or a fee.

(C) 20XX ACM 1529-3785/20XX/0700-0001 $\$ 5.00$ 
Categories and Subject Descriptors: C.2.1 [Computer-Communication Networks]: Network Architecture and Design-Distributed networks; G.1.6 [Numerical Analysis]: OptimizationConstrained optimization, Convex programming, Integer programming, Nonlinear programming; G.3 [Probability and Statistics]: Probability and Statistics-Experimental design

General Terms: Algorithms, Design, Performance

Additional Key Words and Phrases: Sensor network, dynamical system, distributed state estimation, randomly occurring nonlinearity $(\mathrm{RON})$, time delays, stability in the mean square

\section{INTRODUCTION}

Recent advances in network technology and sensor research have led to more and more sensing systems which are integrated through a network. Sensor networks consist of a large number of inexpensive wireless devices (nodes) densely distributed over the region of interest [Brooks et al. 2003; Wang et al. 2009]. Sensor networks have been widely applied in practice such as military sensing, physical security, air traffic control, traffic surveillance, video surveillance, distributed robotics and industrial and manufacturing automation. Though the emerging techniques for sensor network itself have been playing an important role in the research community, the revolution of sensor networks relies on more advances in multidisciplinary research [Xiong et al. 2004]. One of the popular research topics for sensor networks is the distributed filtering or estimation problem that has been attracting growing research interests. For distributed estimation problem, the sensors are arranged in a communication graph configuration and the measurements cannot be sent simultaneously or instantaneously to a base station. Each individual sensor in a sensor network locally estimates the system state from not only its own measurement but also its neighboring sensors' measurements according to the given topology. Different from the traditional central filtering techniques [Liu et al. 2004; Gao and Chen 2007; Shi et al. 2006; Wu et al. 2008; Xiong and Lam 2006; Zhang et al. 2006], an effective distributed estimation algorithm should be capable of handling complicated coupling between the sensor nodes as well as network-induced phenomena such as random communication delays and stochastic disturbances.

Owing to their great application potential in large-scale sensor networks with limited capabilities, the distributed estimation or filtering algorithms have gained a great deal of research attention and a variety of distributed strategies have been proposed. For example, in [Olfati-Saber 2007; Olfati-Saber and Shamma 2005], three distributed Kalman filtering (DKF) algorithms have been developed that can be applied to sensor networks with different observation matrices (sensing models) and with different consensus strategies. In [Speranzon et al. 2008], a distributed estimation algorithm has been proposed for sensor networks, where each node computes its estimate as a weighted sum of its own and its neighbors' measurements, and the weights are adaptively updated to minimize the variance of the estimation error. In [Kim et al. 2008], the multiscale approaches have been employed to accelerate the convergence of decentralized consensus problems, where the consecutive consensus iterations are executed on several scales to achieve fast convergence for networks with poor connectivity. In [Yu et al. 2009], a type of distributed consensus filters has been designed based on the pinning control approach where only a 
small fraction of sensors need to measure the target information. More recently, in [Cattivelli and Sayed 2010a; 2010b], the problem of distributed Kalman filtering and smoothing has been investigated, where a set of nodes is required to estimate the state of a linear dynamic system in a collaborative manner and the information is diffused across the network through a sequence of Kalman iterations and data-aggregation.

In reality, the sensor networks are naturally subject to network-induced complexities such as stochastic disturbances, random communication delays and randomly occurring nonlinearities. Firstly, sensor networks are often deployed in a noisy environment and it is not surprising that the stochastic modeling issue has been of vital importance for distributed estimation problems of sensor networks. Note that the additive white noises have been extensively studied in [Olfati-Saber 2007; Speranzon et al. 2008; Yu et al. 2009; Cattivelli and Sayed 2010a] within a standard Kalman filtering framework, but another important type of stochastic disturbances, namely, multiplicative (Itô-type) noises that are related to the stochastic nature of external fields or boundary conditions, have not received adequate attention [Yu et al. 2009]. Secondly, the time-delay phenomenon in spreading information through sensor networks (complex networks or neural networks) is well known to be ubiquitous because of the limited communication capability [Cao et al. 2008; Song and Cao 2007; Yuan et al. 2006; Liu et al. 2008], and therefore the delay effects have been a focus in the research of sensor networks, see e.g. [Cohen and Kapchits 2009; Bekmezci and Alagöz 2009] where the time-delay is assumed to be deterministic. However, the signal transmission delay over sensor networks is inherently of a random nature dependent on the network circumstances. Thirdly, as is well known, the sensor networks are influenced by additive nonlinear disturbances that are caused by environmental circumstances. Such nonlinear disturbances themselves may experience random abrupt changes due probably to abrupt phenomena such as random failures and changes in node interconnections, which give rise to the so-called randomly occurring nonlinearities (RONs), see [Wang et al. 2009; Wang et al. 2010] for more explanations.

It should be pointed out that, although the distributed state estimation problem for sensor networks has stirred considerable research attention, so far, very little research effort has been paid to the design problem for distributed state estimators under network-induced random phenomena including multiplicative noises, random communication delays and RONs. The lack of the research results is mainly due to the mathematical complexity introduced by the node coupling, random delays and RONs. It is, therefore, the aim of this paper to shorten such a gap by launching a study on distributed state estimation problem in sensor networks with RONs subject to time-delays. Specifically, in this paper, we are interested in the design of distributed estimators that can locally predict the system states, where each sensor communicates with the neighboring sensors according to the given topology in terms of a directed graph. By using a novel Lyapunov functional, several delay-dependent criteria are established under which the estimation error from each sensor is globally asymptotically stable in the mean square. A numerical example is given to verify the designed distributed state estimators.

It is noted that the sensor networks considered in this paper possess a distinguish- 
ing feature that is fundamentally different from other kinds of networked systems such as those studied in [Naghshtabrizi and Hespanha 2005; Yue et al. 2004], i.e., the topology (space) information about the sensor network is now playing a central role. More specifically, each individual sensor needs to estimate the system state based not only on its own measurement but also on its neighboring sensors' measurements according to certain topology. Subsequently, the addressed distributed state estimation problem for sensor networks is fundamentally different from the central state estimation problem for networked systems in problem formulation, estimator structure selection and algorithm development due mainly to the available topology information. The main contributions of this paper are listed as follows. 1) In the plant under consideration, multiplicative (Itô-type) noises and random communication delays exist simultaneously that render more practical significance of our current research. 2) RONs are introduced to describe the phenomena of nonlinear disturbances appearing in a random way. 3) Intensive stochastic analysis is conducted to obtain easily verifiable conditions for the existence of the desired estimators. The rest of the paper is organized as follows. In Section 2, the problem addressed is formulated and some preliminaries are briefly outlined. In Section 3, the main theorems and corollaries are given for the distributed state estimation of the dynamical systems describing the sensor networks. Finally, conclusions are drawn in Section 4.

Notations. The notations used throughout this paper are fairly standard except where otherwise stated. $\mathbb{R}^{n}$ and $\mathbb{R}^{m \times n}$ denote the $n$-dimensional Euclidean space and the set of all $m \times n$ real matrices, respectively. $I_{n}$ and $0_{n}$ represent, respectively, the identity matrix and the zero matrix of dimension $n$. The Kronecker product of matrices $A \in \mathbb{R}^{m \times n}$ and $B \in \mathbb{R}^{p \times q}$ is a matrix in $\mathbb{R}^{m p \times n q}$ and denoted as $A \otimes B$. The notation $X>0$ (respectively, $X \geq 0$ ) for $X \in \mathbb{R}^{n \times n}$ means that matrix $X$ is real, symmetric and positive definite (respectively, positive semi-definite). $\operatorname{diag}(\cdots)$ stands for the block-diagonal matrix and the asterisk ' $*$ ' in a symmetric matrix is used to denote the term that is induced by symmetry. $\operatorname{col}(\cdots)$ denotes a matrix column with blocks given by the matrices in $(\cdots)$. Let $(\Omega, \mathcal{F}, \mathcal{P})$ be a complete probability space with a filtration $\left\{\mathcal{F}_{t}\right\}_{t \geq 0}$ satisfying the usual conditions. $\operatorname{Prob}\{\beta\}$ stands for the occurrence probability of the event $\beta$ and $\mathbb{E}\{\alpha\}$ means the mathematical expectation of the stochastic variable $\alpha$ with respect to the given probability measure $\mathcal{P}$. Matrices, if not stated, are assumed to be compatible for algebraic operations.

\section{PROBLEM FORMULATION AND PRELIMINARIES}

In this paper, our main aim is to estimate the state of a target using a network of sensors. In this context, there are two models to be considered, namely, the model describing the dynamics of the target referred to as the dynamical plant and the model of the sensor network consisting of nodes and topology among the nodes. Let us now start with the dynamical plant described by the following Itô-type stochastic system with RON subject to time-delay:

$$
d s(t)=[A s(t)+B f(t, s(t))+\delta(t) D g(t, s(t-\tau(t)))] d t+M_{0} s(t) d \omega(t),
$$


where $s(t) \in \mathbb{R}^{n}$ is the state vector of the plant; $A, B, D$ and $M_{0}$ are known constant matrices; $\omega(t)$ is a scalar Brownian motion defined on $(\Omega, \mathcal{F}, \mathcal{P})$ with

$$
\mathbb{E}\{d \omega(t)\}=0 \quad \text { and } \quad \mathbb{E}\left\{[d \omega(t)]^{2}\right\}=1 .
$$

$f(t, s(t))$ and $g(t, s(t-\tau(t)))$ are continuous nonlinear functions, and $\tau(t)$ is a known time-varying differentiable function representing the discrete time delay which satisfies

$$
0<\underline{\tau} \leq \tau(t) \leq \bar{\tau}, \quad \dot{\tau}(t) \leq \mu ; \quad\left(\forall t \in \mathbb{R}_{+}\right)
$$

where $\underline{\tau}, \bar{\tau}$ and $\mu$ are constants. Note that the time-delay has long been a focus in systems modeling and control, see e.g. [Lu and Ho 2010; Lu et al. 2009; Meng et al. 2009; Karimi and Gao 2010; Goodall and Postoyan 2010]. Moreover, $\delta(t)$ is a Bernoulli distributed variable defined by

$$
\delta(t)= \begin{cases}1, & \text { if Event } 1 \text { occurs } \\ 0, & \text { if Event } 2 \text { occurs }\end{cases}
$$

in which Event 1 refers to the case that the dynamics of system (1) is affected by the past state $s(t-\tau(t))$ while, if this is not the case, Event 2 occurs. As pointed out in [Yue et al. 2009; Gao et al. 2009; Lin et al. 2009], $\delta(t)$ is a Markovian process. It is also assumed in this paper that the variables $\delta(t)$ and $\omega(t)$ are mutually independent, and $\delta(t)$ follows an exponential distribution of switches with

$$
\operatorname{Prob}\{\delta(t)=1\}=\mathbb{E}\{\delta(t)\}=\delta_{0}, \quad \operatorname{Prob}\{\delta(t)=0\}=1-\mathbb{E}\{\delta(t)\}=1-\delta_{0} ;
$$

where $\delta_{0}\left(0 \leq \delta_{0} \leq 1\right)$ is the known occurrence probability of the event of experiencing the regulating function $g(\cdot, \cdot)$. Obviously, it follows from the above hypothesis that

$$
\mathbb{E}\left\{\delta(t)-\delta_{0}\right\}=0 \quad \text { and } \quad \mathbb{E}\left\{\left(\delta(t)-\delta_{0}\right)^{2}\right\}=\delta_{0}\left(1-\delta_{0}\right) .
$$

REMARK 1. In this paper, the random variable $\delta(t)$ is utilized to model the probability distribution of the nonlinear function describing the regulation effect of the delayed states, i.e., the probability distribution of the randomly occurring nonlinearity (RON) $g(\cdot, \cdot)$. The concept of RON was firstly proposed in [Wang et al. 2009; Wang et al. 2010] to account for the binary switch between two nonlinear functions which might reflect more realistic characteristics in complex networks.

Next, let us deal with the sensor network model. The sensor network is assumed to have $N$ sensors modeled by

$$
y_{i}(t)=C_{i} s(t)+W_{i} h_{i}(t, s(t))+M_{i} s(t) d \omega(t) / d t, \quad i=1,2, \ldots, N
$$

where $y_{i}(t) \in \mathbb{R}^{m}$ is the measurement output observed by sensor $i$ from the plant, $C_{i}, W_{i}$ and $M_{i}$ are known real constant matrices, and $h_{i}(t, s(t))$ denotes the nonlinear deterministic disturbance on the sensor output.

REMARK 2. The dynamical plant modeled according to physical laws is continuoustime by its nature. Continuous-time sensor networks have been a focus of research in the past decade. For example, in [Sinopoli et al. 2003], a hierarchical model has been presented that is composed of continuous time-trigger components at the low level and discrete event-triggered components at the high level. Recently, it has 
been confirmed in [Egea-Lopez et al. 2006] that many necessary models for sensor networks are in the continuous-time domain (e.g., heat transmission, battery discharge). On the other hand, stochastic disturbances exist universally in realworld sensor networks. For instance, the signal transfer through wireless networks could be perturbed randomly from the release of probabilistic causes such as neurotransmitters and packet dropouts. However, the stochastic noise by means of scalar Brownian motion has not been explicitly taken into account for sensor networks in [Sinopoli et al. 2003; Egea-Lopez et al. 2006]. Therefore, one of the objectives of this paper is to include the Brownian motion and randomly occurring nonlinearities in the sensor network model so as to better reflect the engineering practice. Moreover, when it comes to the implementation of continuous-time networks for the sake of computer-based simulation, experimentation, computation or estimation, it is usual to use digital signals from the sensor output to analyze/control/simulate the continuous-time networks. In this case, only the samples of the sensor output signals at discrete time instants are employed, which gives rise to the sampled-data distributed filtering problem as one of the interesting topics to be investigated in the near future. For the present research in this paper, it is assumed that a device of digital-to-analog converter (DAC) exists to convert a digital (usually binary) code to an analog signal (e.g. current, voltage, electric charge) and therefore the sensor model can be regarded as continuous-time.

In this paper, let $\mathscr{G}=(\nu, \varepsilon)$ be the directed graph of order $N$ with nonnegative adjacency matrix $\mathcal{L}=\left[l_{i j}\right]$ that characterizes the interconnection topology of the sensor network. More specifically, $\nu=\{1,2, \ldots, N\}$ represents the set of labeled nodes; $\varepsilon \subseteq \nu \times \nu$ is the set of edges and each edge can be denoted by an ordered pair of vertices $(i, j)$ corresponding to the information transmission from sensor $j$ to sensor $i ; \mathcal{L}=\left[l_{i j}\right]$ is defined as follows: $l_{i j}>0$ if $(i, j) \in \varepsilon, l_{i j}=0$ otherwise. Moreover, we assume $l_{i i}=1$ for all $i \in \nu$, and therefore $(i, i)$ can be regarded as an additional edge. The set of neighbors of node $i \in \nu$ plus the node itself are denoted by $\mathcal{N}_{i}=\{j \in \nu:(i, j) \in \varepsilon\}$. Furthermore, according to the given network topology, it is assumed that each sensor node can receive information from its neighboring nodes in the sensor network. The aim of this paper is to estimate the states of the plant at current times by utilizing the neighboring measurements from the sensor network.

Construct the following state estimator on sensor node $i$ :

$$
\begin{aligned}
d x_{i}(t)= & {\left[A x_{i}(t)+B f\left(t, x_{i}(t)\right)+\delta_{0} D g\left(t, x_{i}(t-\tau(t))\right)\right.} \\
& \left.+\sum_{j \in \mathcal{N}_{i}} l_{i j} K_{i j}\left(y_{j}(t)-C_{j} x_{j}(t)-W_{j} h_{j}\left(t, x_{j}(t)\right)\right)\right] d t
\end{aligned}
$$

where $x_{i}(t) \in \mathbb{R}^{n}$ is the estimation of the state $s(t)$ of dynamical plant based on the node $i$, and $K_{i j} \in \mathbb{R}^{n \times m}$ is the estimator gain matrix to be designed.

REMARK 3. The state estimator in the form (7) reflects the collaborative behavior of the distributed estimation, that is, each individual sensor needs to estimate the system state based not only on its own measurement but also on its neighbor- 
ing sensors' measurements according to the given topology. Note that $\mathcal{N}_{i}=\{j \in$ $\nu:(i, j) \in \varepsilon\}$ denotes the set of neighbors of node $i \in \nu$ plus the node itself.

Throughout this paper, the following assumptions are made on the nonlinear functions in (1) and (6).

Assumption 1. There exist matrices $\Lambda, \Sigma$ and $\Gamma_{i}(i=1,2, \ldots, N)$ such that the following inequalities

$$
\begin{aligned}
\|f(t, x)-f(t, y)\| & \leq\|\Lambda(x-y)\|, \\
\|g(t, x)-g(t, y)\| & \leq\|\Sigma(x-y)\|, \\
\left\|h_{i}(t, x)-h_{i}(t, y)\right\| & \leq\left\|\Gamma_{i}(x-y)\right\|
\end{aligned}
$$

hold for all $t \in \mathbb{R}_{+}$and $x, y \in \mathbb{R}^{n}$.

REMARK 4. Assumption 1 is about the Lipschitz continuity of the nonlinear functions $f, g$ and $h_{i}$. Intuitively, the Lipschitz continuity guarantees that these nonlinear functions are limited in how fast they can change, i.e., for every pair of points on the graph of the functions, their tangent line-segment's slope has absolutevalue no greater than a definite real number. In other words, these nonlinear functions cannot be too steep. With such a Lipschitz continuity assumption on the network parameters, we would be able to deduce the asymptotic stability of the equilibrium point of the error dynamics for the distributed estimation problem, and therefore guarantee the convergence of the state estimation algorithm. Note that the nonlinearities satisfying the Lipschitz conditions are encountered in many engineering systems such as systems with multiplicative noises, systems with nonlinearity dependent on the norm of states, systems with nonlinearity dependent on the absolute value of states, and systems with nonlinearity dependent on the sign of a nonlinear function of states. For example, for the localization problem of Unmanned Aerial Vehicles (UAVs) [Shen et al. 2011] with their movement in a beeline only, the dynamic model of a $U A V$ is usually a nonlinear system containing some monomials satisfying the Lipschitz continuity constraints.

Assumption 2. For all $t \in \mathbb{R}_{+}$, the following equalities hold:

$$
f(t, 0)=0, \quad g(t, 0)=0, \quad h_{i}(t, 0)=0 .
$$

REMARK 5. Assumption 2 is concerned with the existence of the zero equilibrium, which is for the convenience of mathematical analysis. It is known that the existence and uniqueness of the equilibrium for the overall estimation error dynamics can be guaranteed by the Lipschitz continuity of the nonlinear functions $f, g$ and $h_{i}$. Assumption 2 further ensures that such equilibrium is zero, which would facilitate the stability analysis later on. The asymptotic stability of the zero equilibrium means that the state estimation error from each sensor asymptotically tends to zero, thereby guaranteeing the convergence of the proposed distributed filtering algorithm. 
By letting $e_{i}(t)=s(t)-x_{i}(t)$, it follows from (1), (6) and (7) that

$$
\begin{aligned}
d e_{i}(t)= & {\left[A e_{i}(t)+B \tilde{f}\left(t, e_{i}(t)\right)+\delta_{0} D \tilde{g}\left(t, e_{i}(t-\tau(t))\right)\right.} \\
& \left.+\left(\delta(t)-\delta_{0}\right) D g(t, s(t-\tau(t)))-\sum_{j \in \mathcal{N}_{i}} l_{i j} K_{i j}\left(C_{j} e_{j}(t)+W_{j} \tilde{h}_{j}\left(t, e_{j}(t)\right)\right)\right] d t \\
& +\left(M_{0}-\sum_{j \in \mathcal{N}_{i}} l_{i j} K_{i j} M_{j}\right) s(t) d \omega(t)
\end{aligned}
$$

where $i=1,2, \ldots, N ; \tilde{f}\left(t, e_{i}(t)\right)=f(t, s(t))-f\left(t, x_{i}(t)\right), \tilde{g}\left(t, e_{i}(t-\tau(t))\right)=g(t, s(t-$ $\tau(t)))-g\left(t, x_{i}(t-\tau(t))\right)$ and for all $j \in \mathcal{N}_{i}, \tilde{h}_{j}\left(t, e_{j}(t)\right)=h_{j}(t, s(t))-h_{j}\left(t, x_{j}(t)\right)$.

Denoting $e(t)=\operatorname{col}\left(e_{1}(t), e_{2}(t), \ldots, e_{N}(t)\right)$, then the estimation error dynamics for the sensor network can be rewritten in a compact form as

$$
\begin{aligned}
d e(t)= & {\left[\left(I_{N} \otimes A\right) e(t)+\left(I_{N} \otimes B\right) F(t, e(t))+\delta_{0}\left(I_{N} \otimes D\right) G(t, e(t-\tau(t)))\right.} \\
& \left.+\left(\delta(t)-\delta_{0}\right) \tilde{D} g(t, s(t-\tau(t)))-\bar{K} \tilde{C} e(t)-\bar{K} \tilde{W} H(t, e(t))\right] d t \\
& +\left(\tilde{M}_{0}-\bar{K} \tilde{M}\right) s(t) d \omega(t) \\
= & {\left[\left(I_{N} \otimes A-\bar{K} \tilde{C}\right) e(t)+\left(I_{N} \otimes B\right) F(t, e(t))-\bar{K} \tilde{W} H(t, e(t))\right.} \\
& \left.+\delta_{0}\left(I_{N} \otimes D\right) G(t, e(t-\tau(t)))+\left(\delta(t)-\delta_{0}\right) \tilde{D} g(t, s(t-\tau(t)))\right] d t \\
& +\left(\tilde{M}_{0}-\bar{K} \tilde{M}\right) s(t) d \omega(t),
\end{aligned}
$$

where

$$
\begin{gathered}
\tilde{D}=\operatorname{col}(D, D, \ldots, D), \quad \tilde{C}=\operatorname{diag}\left(C_{1}, C_{2}, \ldots, C_{N}\right), \\
\tilde{W}=\operatorname{diag}\left(W_{1}, W_{2}, \ldots, W_{N}\right), \quad \tilde{M}_{0}=\operatorname{col}\left(M_{0}, M_{0}, \ldots, M_{0}\right), \\
\tilde{M}=\operatorname{col}\left(M_{1}, M_{2}, \ldots, M_{N}\right), \quad F(t, e(t))=\left[\begin{array}{c}
\tilde{f}\left(t, e_{1}(t)\right) \\
\tilde{f}\left(t, e_{2}(t)\right) \\
\vdots \\
\tilde{f}\left(t, e_{N}(t)\right)
\end{array}\right], \\
H(t, e(t))=\left[\begin{array}{c}
\tilde{g}\left(t, e_{1}(t-\tau(t))\right) \\
\tilde{g}\left(t, e_{2}(t-\tau(t))\right) \\
\vdots \\
\tilde{h}_{1}\left(t, e_{1}(t)\right) \\
\vdots \\
\tilde{h}_{N}\left(t, e_{N}(t)\right)
\end{array}\right], \quad G(t, e(t-\tau(t)))=\left[\begin{array}{c}
\tilde{g}\left(t, e_{N}(t-\tau(t))\right)
\end{array}\right],
\end{gathered}
$$

and $\bar{K}=\left(l_{i j} K_{i j}\right)_{N \times N}$ is a sparse matrix satisfying $\bar{K} \in \mathscr{W}_{n \times m}$, where $\mathscr{W}_{n \times m}$ is defined as

$$
\mathscr{W}_{n \times m}=\left\{\bar{U}=\left[U_{i j}\right] \in \mathbb{R}^{n N \times m N} \mid U_{i j} \in \mathbb{R}^{n \times m}, U_{i j}=0 \text { if } j \notin \mathcal{N}_{i}\right\} .
$$

In terms of Assumption 1, one can easily obtain

$$
\begin{aligned}
& \|F(t, e(t))\| \leq\|\tilde{\Lambda} e(t)\|, \quad\|H(t, e(t))\| \leq\|\tilde{\Gamma} e(t)\|, \\
& \|G(t, e(t-\tau(t)))\| \leq\|\tilde{\Sigma} e(t-\tau(t))\|
\end{aligned}
$$

ACM Transactions on Sensor Networks, Vol. X, No. X, XX 20XX. 
where $\tilde{\Lambda}=\operatorname{diag}(\Lambda, \Lambda, \ldots, \Lambda), \tilde{\Sigma}=\operatorname{diag}(\Sigma, \Sigma, \ldots, \Sigma)$ and $\tilde{\Gamma}=\operatorname{diag}\left(\Gamma_{1}, \Gamma_{2}, \ldots, \Gamma_{N}\right)$.

Letting $\eta(t)=\left[s^{T}(t), e^{T}(t)\right]^{T}$, the combination of (1) and (9) yields the following augmented system

$$
\begin{aligned}
d \eta(t)= & {\left[\mathcal{A} \eta(t)+\mathcal{B} \mathscr{F}(t, \eta(t))+\delta_{0} \mathcal{D}_{1} \mathcal{G}(t, \eta(t-\tau(t)))\right.} \\
& \left.+\left(\delta(t)-\delta_{0}\right) \mathcal{D}_{2} \mathcal{G}(t, \eta(t-\tau(t)))\right] d t+\mathcal{M} \eta(t) d \omega(t) \\
= & {\left[\mathcal{Y}(t)+\left(\delta(t)-\delta_{0}\right) \mathcal{D}_{2} \mathcal{G}(t, \eta(t-\tau(t)))\right] d t+\mathcal{M} \eta(t) d \omega(t), }
\end{aligned}
$$

where $\mathcal{A}=\operatorname{diag}\left(A, I_{N} \otimes A-\bar{K} \tilde{C}\right), \mathcal{D}_{1}=\operatorname{diag}\left(D, I_{N} \otimes D\right)$,

$$
\begin{aligned}
& \mathcal{B}=\left[\begin{array}{ccc}
B & 0 & 0 \\
0 & I_{N} \otimes B & -\bar{K} \tilde{W}
\end{array}\right], \quad \mathcal{D}_{2}=\left[\begin{array}{ll}
D & 0 \\
\tilde{D} & 0
\end{array}\right], \quad \mathcal{M}=\left[\begin{array}{cc}
M_{0} & 0 \\
\tilde{M}_{0}-\bar{K} \tilde{M} & 0
\end{array}\right], \\
& \mathscr{F}(t, \eta(t))=\left[\begin{array}{l}
f(t, s(t)) \\
F(t, e(t)) \\
H(t, e(t))
\end{array}\right], \quad \mathcal{G}(t, \eta(t-\tau(t)))=\left[\begin{array}{c}
g(t, s(t-\tau(t))) \\
G(t, e(t-\tau(t)))
\end{array}\right] .
\end{aligned}
$$

The initial condition associated with (12) is given as

$$
\eta(\theta)=\varphi(\theta), \quad \theta \in[-\bar{\tau}, 0]
$$

where

$$
\varphi(\cdot) \in L_{\mathcal{F}_{0}}^{2}\left([-\bar{\tau}, 0], \mathbb{R}^{(N+1) n}\right)
$$

and $L_{\mathcal{F}_{0}}^{2}\left([-\bar{\tau}, 0], \mathbb{R}^{(N+1) n}\right)$ is the family of all $\mathcal{F}_{0}$-measurable $C\left([-\bar{\tau}, 0], \mathbb{R}^{(N+1) n}\right)$ valued random variables satisfying $\sup _{-\bar{\tau} \leq \theta \leq 0} \mathbb{E}\left\{\|\varphi(\theta)\|^{2}\right\}<\infty$, and the corresponding solution of (12) is denoted as $\eta(t ; \varphi)$.

Before stating the problem addressed in this paper, the following stability concept for the stochastic system (12) is introduced.

Definition 1. For all $i=1,2, \ldots, N$, the system (7) is said to be a globally asymptotic state estimator on sensor node $i$ of the dynamical system (1) if the zero solution of the augmented system (12) is globally asymptotically stable in the mean square, i.e.,

$$
\lim _{t \rightarrow \infty} \mathbb{E}\left\{\|\eta(t ; \varphi)\|^{2}\right\}=0
$$

holds for all $\varphi(\cdot) \in L_{\mathcal{F}_{0}}^{2}\left([-\bar{\tau}, 0], \mathbb{R}^{(N+1) n}\right)$.

The purpose of the distributed state estimation problem is to seek estimator gain matrices $K_{i j} \in \mathbb{R}^{n \times m}\left(i=1,2, \ldots, N, j \in \mathcal{N}_{i}\right)$ such that the zero solution of the augmented system (12) is globally asymptotically stable in the mean square.

\section{MAIN RESULTS}

Some lemmas are introduced before giving the main results.

Lemma 1. Let $x \in \mathbb{R}^{n}, y \in \mathbb{R}^{n}$ and matrix $Q>0$. Then the inequality $2 x^{T} y \leq$ $x^{T} Q x+y^{T} Q^{-1} y$ holds.

Lemma 2. [Boyd et al. 1994] The following linear matrix inequality

$$
\left[\begin{array}{cc}
Q(x) & S(x) \\
S^{T}(x) & R(x)
\end{array}\right]>0
$$

ACM Transactions on Sensor Networks, Vol. X, No. X, XX 20XX. 
where $Q(x)=Q^{T}(x), R(x)=R^{T}(x)$ and $S(x)$ depend affinely on $x$, is equivalent to one of the following conditions:

(i) $R(x)>0, \quad Q(x)-S(x) R^{-1}(x) S^{T}(x)>0$;

(ii) $Q(x)>0, \quad R(x)-S^{T}(x) Q^{-1}(x) S(x)>0$.

Now, we start to analyze the distributed state estimation problem for the augmented system (12). For this purpose, we have from Assumptions 1-2 that

$$
\|\mathscr{F}(t, \eta(t))\| \leq\|\widehat{\mathbf{\Lambda}} \eta(t)\|, \quad\|\mathcal{G}(t, \eta(t-\tau(t)))\| \leq\|\widehat{\mathbf{\Sigma}} \eta(t-\tau(t))\| ;
$$

where

$$
\widehat{\boldsymbol{\Lambda}}=\left[\begin{array}{cc}
\Lambda & 0 \\
0 & \tilde{\Lambda} \\
0 & \tilde{\Gamma}
\end{array}\right], \quad \widehat{\boldsymbol{\Sigma}}=\left[\begin{array}{cc}
\Sigma & 0 \\
0 & \tilde{\Sigma}
\end{array}\right]
$$

and $\tilde{\Lambda}, \tilde{\Sigma}, \tilde{\Gamma}$ are defined in (11).

Obviously, it follows from (14) that for any positive scalars $\epsilon_{1}$ and $\epsilon_{2}$, the following two inequalities hold:

$$
\epsilon_{1} \eta^{T}(t) \widehat{\boldsymbol{\Lambda}}^{T} \widehat{\boldsymbol{\Lambda}} \eta(t)-\epsilon_{1} \mathscr{F}^{T}(t, \eta(t)) \mathscr{F}(t, \eta(t)) \geq 0 ;
$$

and

$$
\epsilon_{2} \eta^{T}(t-\tau(t)) \widehat{\boldsymbol{\Sigma}}^{T} \widehat{\boldsymbol{\Sigma}} \eta(t-\tau(t))-\epsilon_{2} \mathcal{G}^{T}(t, \eta(t-\tau(t))) \mathcal{G}(t, \eta(t-\tau(t))) \geq 0 .
$$

THEOREM 1. Under Assumptions 1-2, for the given $\bar{K}$, the network in (12) with time-varying delays is globally asymptotically stable in the mean square if there exist matrices $\mathbf{P}>0, \mathbf{Q} \geq 0, \mathbf{R} \geq 0, \mathbf{S}>0, \mathbf{Z}>0$, matrices $\mathbf{T}_{j}(j=1,2,3,4)$ and scalars $\epsilon_{1}>0, \epsilon_{2}>0$ such that

$$
\left[\begin{array}{cc}
\Xi & \Upsilon \\
* & -\mathscr{S}
\end{array}\right]<0
$$

where $\Upsilon=\left[\begin{array}{llllll}\aleph_{1} & \aleph_{1} & \aleph_{2} & \aleph_{2} & \aleph_{3} & \aleph_{3}\end{array}\right], \mathscr{S}=\operatorname{diag}(\mathbf{S}, \mathbf{Z}, \mathbf{S}, \mathbf{Z}, \mathbf{S}, \mathbf{Z})$,

$$
\begin{aligned}
& \Xi=\left[\begin{array}{ccccccc}
\Xi_{11} & \mathbf{T}_{1}^{T} & 0 & 0 & \mathbf{P B} & \delta_{0} \mathbf{P} \mathcal{D}_{1} & \mathcal{A}^{T} \mathbf{T}_{4}^{T} \\
* & -\mathbf{Q}-\mathbf{T}_{1}-\mathbf{T}_{1}^{T} & \mathbf{T}_{2}^{T} & 0 & 0 & 0 & 0 \\
* & * & \Xi_{33} & \mathbf{T}_{3}^{T} & 0 & 0 & 0 \\
* & * & * & -\mathbf{T}_{3}-\mathbf{T}_{3}^{T} & 0 & 0 & 0 \\
* & * & * & * & -\epsilon_{1} I & 0 & \mathcal{B}^{T} \mathbf{T}_{4}^{T} \\
* & * & * & * & * & \Xi_{66} & \delta_{0} \mathcal{D}_{1}^{T} \mathbf{T}_{4}^{T} \\
* & * & * & * & * & * & \bar{\tau} \mathbf{S}-\mathbf{T}_{4}-\mathbf{T}_{4}^{T}
\end{array}\right], \\
& \aleph_{1}=\left[\begin{array}{cccccccc}
0 & \sqrt{\underline{\tau}} \mathbf{T}_{1}^{T} & 0 & 0 & 0 & 0 & 0
\end{array}\right]^{T}, \quad \aleph_{2}=\left[\begin{array}{lllllll}
0 & 0 & \sqrt{\bar{\tau}-\underline{\tau}} \mathbf{T}_{2}^{T} & 0 & 0 & 0 & 0
\end{array}\right]^{T}, \\
& \aleph_{3}=\left[\begin{array}{llllllll}
0 & 0 & 0 & \sqrt{\bar{\tau}-\underline{\tau}} \mathbf{T}_{3}^{T} & 0 & 0 & 0
\end{array}\right]^{T}, \quad \Xi_{66}=\bar{\tau} \delta_{0}\left(1-\delta_{0}\right) \mathcal{D}_{2}^{T} \mathbf{Z} \mathcal{D}_{2}-\epsilon_{2} I, \\
& \Xi_{11}=\mathbf{P} \mathcal{A}+\mathcal{A}^{T} \mathbf{P}+\mathcal{M}^{T} \mathbf{P} \mathcal{M}+\mathbf{Q}+\mathbf{R}+\epsilon_{1} \widehat{\boldsymbol{\Lambda}}^{T} \widehat{\boldsymbol{\Lambda}}, \\
& \Xi_{33}=-(1-\mu) \mathbf{R}-\mathbf{T}_{2}-\mathbf{T}_{2}^{T}+\epsilon_{2} \widehat{\boldsymbol{\Sigma}}^{T} \widehat{\boldsymbol{\Sigma}} \text {. }
\end{aligned}
$$

Proof. See Appendix 1.

REMARK 6. In Theorem 1, the Lyapunov-Krasovskii functional approach is employed to establish delay-dependent criterion that ensures the network in (12) with ACM Transactions on Sensor Networks, Vol. X, No. X, XX 20XX. 
time-varying delays to be globally asymptotically stable in the mean square, in other words, the distributed estimator exists. The criterion is expressed in terms of the solution to certain linear matrix inequalities (LMIs) that can be effectively solved and checked by the algorithms such as the interior-point method in the Matlab Toolbox. Note that the proposed Lyapunov-Krasovskii functional (25) is novel that caters the "energy" involving the time-delay, the lower bound of the time-delay, the upper bound of the time-delay, as well as the mathematical expectation of the stochastic variable. The existence of the decision variables $(\mathbf{P}>0, \mathbf{Q} \geq 0, \mathbf{R} \geq 0, \mathbf{S}>0$, $\mathbf{Z}>0$ ) in (17) implies the existence of the Lyapunov-Krasovskii functional (25), where these decision variables do have their physical meaning. For example, the positive definite matrix $\mathbf{P}>0$ is associated with the energy of the network state and $\mathbf{Q} \geq 0$ is related to the energy of the network state on the delayed interval. If (17) holds, the derivative of the Lyapunov-Krasovskii functional along the network trajectory is less than zero. This means the total energy of the whole network monotonically decreases when time goes, which in turn implies the asymptotic stability of the network.

To derive the design scheme for state estimator parameters, we still need to introduce the following lemma whose proof is straightforward and therefore omitted here.

LEMmA 3. Let $\mathbb{P}=\operatorname{diag}\left(\mathbb{P}_{11}, \mathbb{P}_{22}, \ldots, \mathbb{P}_{N N}\right)$ with $\mathbb{P}_{i i} \in \mathbb{R}^{n \times n}(i=1,2, \ldots, N)$ being invertible matrices. If $X=\mathbb{P} \bar{U}$ for $\bar{U} \in \mathbb{R}^{n N \times m N}$, then we have $\bar{U} \in \mathscr{W}_{n \times m} \Leftrightarrow$ $X \in \mathscr{W}_{n \times m}$.

Based on Lemma 3, we can obtain the following theorem which shows that the distributed state estimation problem can be solved if the solution of a matrix inequality exists.

THEOREM 2. Under Assumptions 1-2, the system (7) is a globally asymptotic state estimator on sensor node $i(i=1,2, \ldots, N)$ of the dynamical system (1) if there exist matrices $P_{k k}>0(k=1,2, \ldots, N+1), \mathbf{Q} \geq 0, \mathbf{R} \geq 0, \mathbf{S}>0, \mathbf{Z}>0$, matrices $T_{41}, T_{43}, \mathbf{T}_{j}(j=1,2,3), X \in \mathscr{W}_{n \times m}$ and scalars $\epsilon_{1}>0, \epsilon_{2}>0$ such that

$$
\left[\begin{array}{cc}
\Pi & \widehat{\Upsilon} \\
* & -\widehat{\mathscr{S}}
\end{array}\right]<0,
$$

where $\widehat{\Upsilon}=\left[\begin{array}{lllllll}\aleph_{1} & \aleph_{1} & \aleph_{2} & \aleph_{2} & \aleph_{3} & \aleph_{3} & \aleph_{4}\end{array}\right], \widehat{\mathscr{S}}=\operatorname{diag}\left(\mathbf{S}, \mathbf{Z}, \mathbf{S}, \mathbf{Z}, \mathbf{S}, \mathbf{Z}, \mathbf{P}_{22}\right), \mathbf{P}_{22}=$ $\operatorname{diag}\left(P_{22}, P_{33}, \ldots, P_{N+1, N+1}\right)$,

$\aleph_{4}=\left[\begin{array}{c}\Pi_{1,10} \\ 0 \\ 0 \\ 0 \\ 0 \\ 0 \\ 0\end{array}\right], \Pi=\left[\begin{array}{ccccccc}\Pi_{11} & \mathbf{T}_{1}^{T} & 0 & 0 & \Pi_{15} & \delta_{0} \Pi_{16} & \Pi_{17} \\ * & -\mathbf{Q}-\mathbf{T}_{1}-\mathbf{T}_{1}^{T} & \mathbf{T}_{2}^{T} & 0 & 0 & 0 & 0 \\ * & * & \Xi_{33} & \mathbf{T}_{3}^{T} & 0 & 0 & 0 \\ * & * & * & -\mathbf{T}_{3}-\mathbf{T}_{3}^{T} & 0 & 0 & 0 \\ * & * & * & * & -\epsilon_{1} I & 0 & \Pi_{57} \\ * & * & * & * & * & \Xi_{66} & \delta_{0} \Pi_{67} \\ * & * & * & * & * & * & \bar{\tau}-\Pi_{77}\end{array}\right]$,

$\aleph_{1}, \aleph_{2}, \aleph_{3}, \Xi_{33}$ and $\Xi_{66}$ are the same as those defined in Theorem $1, \Pi_{11}=\Pi_{11}^{\prime}+$ $\mathbf{Q}+\mathbf{R}+\epsilon_{1} \widehat{\boldsymbol{\Lambda}}^{T} \widehat{\boldsymbol{\Lambda}}$ and $\Pi_{11}^{\prime}=\operatorname{diag}\left(P_{11} A+A^{T} P_{11}+M_{0}^{T} P_{11} M_{0}, \mathbf{P}_{22}\left(I_{N} \otimes A\right)+\left(I_{N} \otimes\right.\right.$ 


$$
\begin{aligned}
& \left.\left.A^{T}\right) \mathbf{P}_{22}-X \tilde{C}-\tilde{C}^{T} X^{T}\right), \\
& \Pi_{15}=\left[\begin{array}{ccc}
P_{11} B & 0 & 0 \\
0 & \mathbf{P}_{22}\left(I_{N} \otimes B\right) & -X \tilde{W}
\end{array}\right], \quad \Pi_{16}=\left[\begin{array}{cc}
P_{11} D & 0 \\
0 & \mathbf{P}_{22}\left(I_{N} \otimes D\right)
\end{array}\right], \\
& \Pi_{17}=\left[\begin{array}{cc}
A^{T} T_{41}^{T} & A^{T} T_{43}^{T} \\
0 & \left(I_{N} \otimes A^{T}\right) \mathbf{P}_{22}-\tilde{C}^{T} X^{T}
\end{array}\right], \quad \Pi_{1,10}=\left[\begin{array}{c}
\tilde{M}_{0}^{T} \mathbf{P}_{22}-\tilde{M}^{T} X^{T} \\
0
\end{array}\right], \\
& \Pi_{57}=\left[\begin{array}{cc}
B^{T} T_{41}^{T} & B^{T} T_{43}^{T} \\
0 & \left(I_{N} \otimes B^{T}\right) \mathbf{P}_{22} \\
0 & -\tilde{W}^{T} X^{T}
\end{array}\right], \Pi_{67}=\left[\begin{array}{cc}
D^{T} T_{41}^{T} & D^{T} T_{43}^{T} \\
0 & \left(I_{N} \otimes D^{T}\right) \mathbf{P}_{22}
\end{array}\right], \\
& \Pi_{77}=\left[\begin{array}{cc}
T_{41}+T_{41}^{T} & T_{43}^{T} \\
T_{43} & 2 \mathbf{P}_{22}
\end{array}\right] \text {. }
\end{aligned}
$$

Moreover,

$$
\bar{K}=\mathbf{P}_{22}^{-1} X
$$

accordingly, parameters $K_{i j}\left(i=1,2, \ldots, N, j \in \mathcal{N}_{i}\right)$ can be derived from (10).

Proof. See Appendix 2.

REMARK 7. In Theorem 1, by taking advantage of the stochastic analysis and the free-weighting matrix technique, the feasibility of the distributed state estimation problem is investigated and several sufficient criteria are derived in the form of matrix inequalities. These sufficient criteria ensure that the desired distributed estimator exists. Based on the results in Theorem 1, the explicit expression of the estimator gains is further derived in Theorem 2 which shows that the distributed state estimation problem can be solved if the solution of a matrix inequality exists. Note that restricting attention to diagonal Lyapunov matrices in order to use matrix inequalities for synthesizing the decentralized control law is well known [Zečević and Siljak 2004]. Furthermore, such inequalities can be easily computed and effectively checked in practice by resorting to the Matlab Control Toolbox.

In the following, two special cases are considered.

Case 1. When there is no RON in the dynamical plant, then equation (1) reduces to

$$
d s(t)=[A s(t)+B f(t, s(t))] d t+M_{0} s(t) d \omega(t) .
$$

By taking the same full-order distributed state estimator (7) with $\delta_{0}=0$, along the similar proof lines of Theorem 2, one has the following result immediately.

Corollary 1. Under Assumptions 1-2, the system (7) with $\delta_{0}=0$ is a globally asymptotic state estimator on sensor node $i(i=1,2, \ldots, N)$ of the dynamical system (20) if there exist matrices $P_{k k}>0(k=1,2, \ldots, N+1)$, matrix $X \in \mathscr{W}_{n \times m}$ and a scalar $\epsilon>0$ such that

$$
\left[\begin{array}{ccc}
\Pi_{11}^{\prime}+\epsilon \widehat{\boldsymbol{\Lambda}}^{T} \widehat{\boldsymbol{\Lambda}} & \Pi_{15} & \Pi_{1,10} \\
* & -\epsilon I & 0 \\
* & * & -\mathbf{P}_{22}
\end{array}\right]<0,
$$

ACM Transactions on Sensor Networks, Vol. X, No. X, XX 20XX. 
where $\Pi_{11}^{\prime}, \Pi_{15}$ and $\Pi_{1,10}$ are the same as defined in Theorem 2. Moreover, $\bar{K}=$ $\mathbf{P}_{22}^{-1} X$; accordingly, parameters $K_{i j}\left(i=1,2, \ldots, N, j \in \mathcal{N}_{i}\right)$ can be derived from (10).

Case 2. When the time-delay in the dynamical system (1) is time-invariant, i.e., $\tau(t) \equiv \tau$, it turns into the following equation:

$$
d s(t)=[A s(t)+B f(t, s(t))+\delta(t) D g(t, s(t-\tau))] d t+M_{0} s(t) d \omega(t) .
$$

With the similar proof as for Theorem 2, we easily obtain the following corollary.

Corollary 2. Under Assumptions 1-2, the system (7) with $\tau(t) \equiv \tau$ is a globally asymptotic state estimator on sensor node $i(i=1,2, \ldots, N)$ of the dynamical system (22) if there exist matrices $P_{k k}>0(k=1,2, \ldots, N+1), \mathbf{Q} \geq 0, \mathbf{S}>0$, $\mathbf{Z}>0$, matrices $T_{41}, T_{43}, \mathbf{T}, X \in \mathscr{W}_{n \times m}$ and scalars $\epsilon_{1}>0, \epsilon_{2}>0$ such that

$$
\left[\begin{array}{cccccccc}
\check{\Pi}_{11} & \mathbf{T}^{T} & \Pi_{15} & \delta_{0} \Pi_{16} & \Pi_{17} & 0 & 0 & \Pi_{1,10} \\
* & \Xi_{22} & 0 & 0 & 0 & \sqrt{\tau} \mathbf{T} & \sqrt{\tau} \mathbf{T} & 0 \\
* & * & -\epsilon_{1} I & 0 & \Pi_{57} & 0 & 0 & 0 \\
* & * & * & \check{\Xi}_{66} & \delta_{0} \Pi_{67} & 0 & 0 & 0 \\
* & * & * & * & \tau \mathbf{S}-\Pi_{77} & 0 & 0 & 0 \\
* & * & * & * & * & -\mathbf{S} & 0 & 0 \\
* & * & * & * & * & * & -\mathbf{Z} & 0 \\
* & * & * & * & * & * & * & -\mathbf{P}_{22}
\end{array}\right]<0
$$

where $\check{\Pi}_{11}=\Pi_{11}^{\prime}+\mathbf{Q}+\epsilon_{1} \widehat{\boldsymbol{\Lambda}}^{T} \widehat{\boldsymbol{\Lambda}}, \check{\Xi}_{22}=-\mathbf{Q}-\mathbf{T}-\mathbf{T}^{T}, \check{\Xi}_{66}=\tau \delta_{0}\left(1-\delta_{0}\right) \mathcal{D}_{2}^{T} \mathbf{Z} \mathcal{D}_{2}-\epsilon_{2} I$ and the other symbols are the same as defined in Theorem 2. Moreover, $\bar{K}=\mathbf{P}_{22}^{-1} X$; accordingly, parameters $K_{i j}\left(i=1,2, \ldots, N, j \in \mathcal{N}_{i}\right)$ can be derived from (10).

REMarK 8. In Refs. [Mou et al. 2008; Mou et al. 2008; Wang et al. 2008], delay fractioning method was utilized to analyze the stability/synchronization for neural/complex networks. The advantage of such method lies in that it fractionalizes the time-delay to arbitrary uniform parts to reduce the conservatism by introducing more matrix variables. Here, one may also employ this technique to reduce the conservatism of the results obtained in Theorem 2. Also, the idea of diffusion strategies of [Cattivelli and Sayed 2009; 2008; Cattivelli et al. 2008; Cattivelli and Sayed 2010a] is to deal with the distributed Kalman filtering and smoothing where a set of nodes is required to estimate the state of a linear dynamic system in a collaborative manner and the information is diffused across the network through a sequence of Kalman iterations and data-aggregation. The diffusion strategies apply mainly to discrete-time case for facilitating the Kalman iterations. Nevertheless, it would be indeed interesting to investigate the possibility of using diffusion strategies and global cost function in the sampled-data distributed filtering problem we are currently looking into.

REMARK 9. Apart from the novelty in the proposed model and addressed research problem, we would also like to take this opportunity to list the essential technical difficulties we have encountered in our research as comparing to the literature on networked estimation problem. 1) The Brownian motion appears in both the dynamical plant and the sensors, and this makes the whole system a set of Itô-type 
stochastic differential equations. The corresponding analysis would have to be carried out by extensive use of the Itô differential role (see (30)), which is non-trivial. 2) Randomly occurring nonlinearities (RONs) and time-delays are introduced to describe the phenomena of nonlinear disturbances and delays appearing in a random way. It is quite subtle to choose the structure of the distributed estimator since the randomly variables should not be present in the estimator and the traditional Luenberger-type estimator is no longer valid. In this paper, we have included the mathematical expectations of the stochastic variables in the estimator structure (see (7)), which brings significant difficulties in ensuring the unbiasedness of the estimation errors. 3) Due to the possibly large size of the sensor networks, the overall error dynamics is governed by a system of dimension $n \times N$, where $n$ is the dimension of the dynamical plant and $N$ is the number of sensors. To cope with such an analysis problem of large dimension, we have chosen to use Kronecker product to describe the system in a more compact form. Nevertheless, calculation over Kronecker product demands extreme care and some special inequalities would need to be developed to handle the time-delays appearing in the Kronecker product. 4) The proposed Lyapunov-Krasovskii functional (25) is novel that caters the "energy" involving the time-delay, the lower bound of the time-delay, the upper bound of the time-delay, as well as the mathematical expectation of the stochastic variable. The selection of such a Lyapunov-Krasovskii functional would largely reduce the conservatism brought from the time-delay at the expense of complicating the derivation. Though we don't regard this as one of our main contributions, as far as we know, the proposed Lyapunov-Krasovskii functional is new and comprehensive that renders the derivation of our main results non-trivial.

\section{CONCLUSIONS}

In this paper, the distributed state estimation problem has been investigated on the sensor network for the dynamical plant system. Both the plant system and each sensor network system are disturbed by external noises described by Brownian motions. Furthermore, RON has been introduced in the dynamical plant to reflect the random effect of the delayed states on the current ones. By using the Lyapunov functional method combing with the stochastic analysis technique, several sufficient criteria have been derived to ensure the state estimation error system to be globally asymptotically stable in the mean square, i.e., the distributed state estimators can be designed to estimate the states of the dynamical plant in a distributed way by utilizing the sensor network output measurements.

\section{Appendix 1: Proof of Theorem 1}

Lemma 2 ensures that inequality (17) is equivalent to $\Xi^{\prime}<0$, where

$$
\Xi^{\prime}=\left[\begin{array}{ccccccc}
\Xi_{11} & \mathbf{T}_{1}^{T} & 0 & 0 & \mathbf{P B} & \delta_{0} \mathbf{P} \mathcal{D}_{1} & \mathcal{A}^{T} \mathbf{T}_{4}^{T} \\
* & \Xi_{22}^{\prime} & \mathbf{T}_{2}^{T} & 0 & 0 & 0 & 0 \\
* & * & \Xi_{33}^{\prime} & \mathbf{T}_{3}^{T} & 0 & 0 & 0 \\
* & * & * & \Xi_{44}^{\prime} & 0 & 0 & 0 \\
* & * & * & * & -\epsilon_{1} I & 0 & \mathcal{B}^{T} \mathbf{T}_{4}^{T} \\
* & * & * & * & * & \Xi_{66} & \delta_{0} \mathcal{D}_{1}^{T} \mathbf{T}_{4}^{T} \\
* & * & * & * & * & * & \bar{\tau} \mathbf{S}-\mathbf{T}_{4}-\mathbf{T}_{4}^{T}
\end{array}\right]
$$

ACM Transactions on Sensor Networks, Vol. X, No. X, XX 20XX. 
and

$$
\begin{aligned}
& \Xi_{22}^{\prime}=-\mathbf{Q}-\mathbf{T}_{1}-\mathbf{T}_{1}^{T}+\underline{\tau} \mathbf{T}_{1}\left(\mathbf{S}^{-1}+\mathbf{Z}^{-1}\right) \mathbf{T}_{1}^{T} \\
& \Xi_{33}^{\prime}=\Xi_{33}+(\bar{\tau}-\underline{\tau}) \mathbf{T}_{2}\left(\mathbf{S}^{-1}+\mathbf{Z}^{-1}\right) \mathbf{T}_{2}^{T}, \\
& \Xi_{44}^{\prime}=-\mathbf{T}_{3}-\mathbf{T}_{3}^{T}+(\bar{\tau}-\underline{\tau}) \mathbf{T}_{3}\left(\mathbf{S}^{-1}+\mathbf{Z}^{-1}\right) \mathbf{T}_{3}^{T} .
\end{aligned}
$$

Consider the Lyapunov-Krasovskii functional candidate as follows:

$$
V\left(t, \eta_{t}\right)=V_{1}\left(t, \eta_{t}\right)+V_{2}\left(t, \eta_{t}\right)+V_{3}\left(t, \eta_{t}\right)+V_{4}\left(t, \eta_{t}\right)+V_{5}\left(t, \eta_{t}\right)
$$

where $\eta_{t}=\eta(t+\theta), \theta \in[-\bar{\tau}, 0]$ and

$$
\begin{aligned}
& V_{1}\left(t, \eta_{t}\right)=\eta^{T}(t) \mathbf{P} \eta(t), \quad V_{2}\left(t, \eta_{t}\right)=\int_{t-\underline{\tau}}^{t} \eta^{T}(\theta) \mathbf{Q} \eta(\theta) d \theta \\
& V_{3}\left(t, \eta_{t}\right)=\int_{t-\tau(t)}^{t} \eta^{T}(\theta) \mathbf{R} \eta(\theta) d \theta, \quad V_{4}\left(t, \eta_{t}\right)=\int_{t-\bar{\tau}}^{t} d v \int_{v}^{t} \mathcal{Y}^{T}(\theta) \mathbf{S} \mathcal{Y}(\theta) d \theta \\
& V_{5}\left(t, \eta_{t}\right)=\delta_{0}\left(1-\delta_{0}\right) \int_{t-\bar{\tau}}^{t} d v \int_{v}^{t} \mathcal{G}^{T}(\theta, \eta(\theta-\tau(\theta))) \mathcal{D}_{2}^{T} \mathbf{Z} \mathcal{D}_{2} \mathcal{G}(\theta, \eta(\theta-\tau(\theta))) d \theta
\end{aligned}
$$

in which $\mathbf{P}>0, \mathbf{Q} \geq 0, \mathbf{R} \geq 0, \mathbf{S}>0$ and $\mathbf{Z}>0$ are matrices to be determined.

From Itô formula [Mao et al. 1998; Mao 1997], one obtains the following stochastic differential:

$$
d V\left(t, \eta_{t}\right)=\mathscr{L} V\left(t, \eta_{t}\right) d t+V_{\eta}\left(t, \eta_{t}\right) \mathcal{M} \eta(t) d \omega(t)
$$

where $\mathscr{L}$ is the infinitesimal operator of $V\left(t, \eta_{t}\right)$ computed as follows:

$$
\begin{aligned}
\mathscr{L} V\left(t, \eta_{t}\right)= & V_{t}\left(t, \eta_{t}\right)+V_{\eta}\left(t, \eta_{t}\right)\left[\mathcal{Y}(t)+\left(\delta(t)-\delta_{0}\right) \mathcal{D}_{2} \mathcal{G}(t, \eta(t-\tau(t)))\right] \\
& +\frac{1}{2} \eta^{T}(t) \mathcal{M}^{T} V_{\eta \eta}\left(t, \eta_{t}\right) \mathcal{M} \eta(t) .
\end{aligned}
$$

Here, $V_{t}\left(t, \eta_{t}\right)$ is the first-order derivative of the real-value function $V\left(t, \eta_{t}\right)$ with respect to $t, V_{\eta}\left(t, \eta_{t}\right)$ and $V_{\eta \eta}\left(t, \eta_{t}\right)$ are, respectively, the first- and second-order derivatives of $V\left(t, \eta_{t}\right)$ with respect to the vector $\eta \in \mathbb{R}^{(N+1) n}$. 
It follows from (25)-(30) that

$$
\begin{aligned}
\mathscr{L} V_{1}\left(t, \eta_{t}\right)= & 2 \eta^{T}(t) \mathbf{P} \mathcal{Y}(t)+2\left(\delta(t)-\delta_{0}\right) \eta^{T}(t) \mathbf{P} \mathcal{D}_{2} \mathcal{G}(t, \eta(t-\tau(t))) \\
& +\eta^{T}(t) \mathcal{M}^{T} \mathbf{P} \mathcal{M} \eta(t) \\
= & 2 \eta^{T}(t) \mathbf{P}\left[\mathcal{A} \eta(t)+\mathcal{B} \mathscr{F}(t, \eta(t))+\delta_{0} \mathcal{D}_{1} \mathcal{G}(t, \eta(t-\tau(t)))\right] \\
& +2\left(\delta(t)-\delta_{0}\right) \eta^{T}(t) \mathbf{P} \mathcal{D}_{2} \mathcal{G}(t, \eta(t-\tau(t)))+\eta^{T}(t) \mathcal{M}^{T} \mathbf{P} \mathcal{M} \eta(t) \\
= & \eta^{T}(t)\left[\mathbf{P} \mathcal{A}+\mathcal{A}^{T} \mathbf{P}+\mathcal{M}^{T} \mathbf{P} \mathcal{M}\right] \eta(t)+2 \eta^{T}(t) \mathbf{P} \mathcal{B} \mathscr{F}(t, \eta(t)) \\
& +2 \delta_{0} \eta^{T}(t) \mathbf{P} \mathcal{D}_{1} \mathcal{G}(t, \eta(t-\tau(t))) \\
& +2\left(\delta(t)-\delta_{0}\right) \eta^{T}(t) \mathbf{P} \mathcal{D}_{2} \mathcal{G}(t, \eta(t-\tau(t))), \\
\mathscr{L} V_{2}\left(t, \eta_{t}\right)= & \eta^{T}(t) \mathbf{Q} \eta(t)-\eta^{T}(t-\underline{\tau}) \mathbf{Q} \eta(t-\underline{\tau}), \\
\mathscr{L} V_{3}\left(t, \eta_{t}\right)= & \eta^{T}(t) \mathbf{R} \eta(t)-(1-\dot{\tau}(t)) \eta^{T}(t-\tau(t)) \mathbf{R} \eta(t-\tau(t)) \\
\leq & \eta^{T}(t) \mathbf{R} \eta(t)-(1-\mu) \eta^{T}(t-\tau(t)) \mathbf{R} \eta(t-\tau(t)), \\
\mathscr{L} V_{4}\left(t, \eta_{t}\right)= & \bar{\tau} \mathcal{Y}^{T}(t) \mathbf{S} \mathcal{Y}(t)-\left(\int_{t-\bar{\tau}}^{t-\tau(t)}+\int_{t-\tau(t)}^{t-\tau}+\int_{t-\underline{\tau}}^{t}\right) \mathcal{Y}^{T}(\theta) \mathcal{S Y}(\theta) d \theta \\
\mathscr{L} V_{5}\left(t, \eta_{t}\right)= & \bar{\tau} \delta_{0}\left(1-\delta_{0}\right) \mathcal{G}^{T}(t, \eta(t-\tau(t))) \mathcal{D}_{2}^{T} \mathbf{Z} \mathcal{D}_{2} \mathcal{G}(t, \eta(t-\tau(t))) \\
& -\delta_{0}\left(1-\delta_{0}\right)\left(\int_{t-\bar{\tau}}^{t-\tau(t)}+\int_{t-\tau(t)}^{t-\tau}+\int_{t-\underline{\tau}}^{t}\right) \mathcal{G}^{T}(\theta, \eta(\theta-\tau(\theta))) \\
& \quad \times \mathcal{D}_{2}^{T} \mathbf{Z} \mathcal{D}_{2} \mathcal{G}(\theta, \eta(\theta-\tau(\theta))) d \theta .
\end{aligned}
$$

For any matrices $\mathbf{T}_{j}(j=1,2,3,4)$ with appropriate dimensions, it follows from the definition of $\mathcal{Y}(t)$ that

$$
\begin{aligned}
& 2 \eta^{T}(t-\underline{\tau}) \mathbf{T}_{1}\left[\eta(t)-\eta(t-\underline{\tau})-\int_{t-\underline{\tau}}^{t} d \eta(\theta)\right]=0, \\
& 2 \eta^{T}(t-\tau(t)) \mathbf{T}_{2}\left[\eta(t-\underline{\tau})-\eta(t-\tau(t))-\int_{t-\tau(t)}^{t-\underline{\tau}} d \eta(\theta)\right]=0, \\
& 2 \eta^{T}(t-\bar{\tau}) \mathbf{T}_{3}\left[\eta(t-\tau(t))-\eta(t-\bar{\tau})-\int_{t-\bar{\tau}}^{t-\tau(t)} d \eta(\theta)\right]=0, \\
& 2 \mathcal{Y}^{T}(t) \mathbf{T}_{4}\left[\mathcal{A} \eta(t)+\mathcal{B} \mathscr{F}(t, \eta(t))+\delta_{0} \mathcal{D}_{1} \mathcal{G}(t, \eta(t-\tau(t)))-\mathcal{Y}(t)\right]=0 .
\end{aligned}
$$

By resorting to Lemma 1, we have

$$
\begin{aligned}
& -2 \eta^{T}(t-\underline{\tau}) \mathbf{T}_{1} \int_{t-\underline{\tau}}^{t} d \eta(\theta) \\
= & -2 \eta^{T}(t-\underline{\tau}) \mathbf{T}_{1} \int_{t-\underline{\tau}}^{t} \mathcal{Y}(\theta) d \theta-2 \eta^{T}(t-\underline{\tau}) \mathbf{T}_{1} \\
& \times \int_{t-\underline{\tau}}^{t}\left(\delta(\theta)-\delta_{0}\right) \mathcal{D}_{2} \mathcal{G}(\theta, \eta(\theta-\tau(\theta))) d \theta-2 \eta^{T}(t-\underline{\tau}) \mathbf{T}_{1} \int_{t-\underline{\tau}}^{t} \mathcal{M} \eta(\theta) d \omega(\theta)
\end{aligned}
$$

ACM Transactions on Sensor Networks, Vol. X, No. X, XX 20XX. 


$$
\begin{aligned}
\leq & \underline{\tau} \eta^{T}(t-\underline{\tau}) \mathbf{T}_{1}\left(\mathbf{S}^{-1}+\mathbf{Z}^{-1}\right) \mathbf{T}_{1}^{T} \eta(t-\underline{\tau})+\int_{t-\underline{\tau}}^{t} \mathcal{Y}^{T}(\theta) \mathbf{S} \mathcal{Y}(\theta) d \theta \\
& +\int_{t-\underline{\tau}}^{t}\left(\delta(\theta)-\delta_{0}\right)^{2} \mathcal{G}^{T}(\theta, \eta(\theta-\tau(\theta))) \mathcal{D}_{2}^{T} \mathbf{Z} \mathcal{D}_{2} \mathcal{G}(\theta, \eta(\theta-\tau(\theta))) d \theta \\
& -2 \eta^{T}(t-\underline{\tau}) \mathbf{T}_{1} \int_{t-\underline{\tau}}^{t} \mathcal{M} \eta(\theta) d \omega(\theta),
\end{aligned}
$$

Similarly, one has

$$
\begin{aligned}
& -2 \eta^{T}(t-\tau(t)) \mathbf{T}_{2} \int_{t-\tau(t)}^{t-\underline{\tau}} d \eta(\theta) \\
\leq & (\tau(t)-\underline{\tau}) \eta^{T}(t-\tau(t)) \mathbf{T}_{2}\left(\mathbf{S}^{-1}+\mathbf{Z}^{-1}\right) \mathbf{T}_{2}^{T} \eta(t-\tau(t)) \\
& +\int_{t-\tau(t)}^{t-\underline{\tau}}\left(\delta(\theta)-\delta_{0}\right)^{2} \mathcal{G}^{T}(\theta, \eta(\theta-\tau(\theta))) \mathcal{D}_{2}^{T} \mathbf{Z} \mathcal{D}_{2} \mathcal{G}(\theta, \eta(\theta-\tau(\theta))) d \theta \\
& +\int_{t-\tau(t)}^{t-\underline{\tau}} \mathcal{Y}^{T}(\theta) \mathbf{S} \mathcal{Y}(\theta) d \theta \\
& -2 \eta^{T}(t-\tau(t)) \mathbf{T}_{2} \int_{t-\tau(t)}^{t-\underline{\tau}} \mathcal{M} \eta(\theta) d \omega(\theta),
\end{aligned}
$$

and

$$
\begin{aligned}
& -2 \eta^{T}(t-\bar{\tau}) \mathbf{T}_{3} \int_{t-\bar{\tau}}^{t-\tau(t)} d \eta(\theta) \\
\leq & (\bar{\tau}-\tau(t)) \eta^{T}(t-\bar{\tau}) \mathbf{T}_{3}\left(\mathbf{S}^{-1}+\mathbf{Z}^{-1}\right) \mathbf{T}_{3}^{T} \eta(t-\bar{\tau}) \\
& +\int_{t-\bar{\tau}}^{t-\tau(t)}\left(\delta(\theta)-\delta_{0}\right)^{2} \mathcal{G}^{T}(\theta, \eta(\theta-\tau(\theta))) \mathcal{D}_{2}^{T} \mathbf{Z} \mathcal{D}_{2} \mathcal{G}(\theta, \eta(\theta-\tau(\theta))) d \theta \\
& +\int_{t-\bar{\tau}}^{t-\tau(t)} \mathcal{Y}^{T}(\theta) \mathbf{S} \mathcal{Y}(\theta) d \theta-2 \eta^{T}(t-\bar{\tau}) \mathbf{T}_{3} \int_{t-\bar{\tau}}^{t-\tau(t)} \mathcal{M} \eta(\theta) d \omega(\theta) .
\end{aligned}
$$

Observing the facts $\tau(t)-\underline{\tau} \leq \bar{\tau}-\underline{\tau}$ and $\bar{\tau}-\tau(t) \leq \bar{\tau}-\underline{\tau}$, and combining with (15), (16) and (31)-(42), we can obtain that

$$
\mathbb{E}\left\{\mathscr{L} V\left(t, \eta_{t}\right)\right\} \leq \mathbb{E}\left\{\xi^{T}(t) \Xi^{\prime} \xi(t)\right\}
$$

where

$\xi(t)=\left[\eta^{T}(t), \eta^{T}(t-\underline{\tau}), \eta^{T}(t-\tau(t)), \eta^{T}(t-\bar{\tau}), \mathscr{F}^{T}(t, \eta(t)), \mathcal{G}^{T}(t, \eta(t-\tau(t))), \mathcal{Y}^{T}(t)\right]^{T}$ and $\Xi^{\prime}$ is defined in (24). This indicates from the Lyapunov stability theory [Schuss 1980] that the dynamics of the augmented system (12) is globally asymptotically stable in the mean square. The proof is complete.

\section{Appendix 2: Proof of Theorem 2}

Noticing that matrix $\bar{K}$ contains all the desired state estimator parameters, inspired by the structure of $\mathcal{A}$, we choose $\mathbf{P}=\operatorname{diag}\left(P_{11}, \mathbf{P}_{22}\right)$, where $P_{11} \in \mathbb{R}^{n \times n}$ and 
$\mathbf{P}_{22} \in \mathbb{R}^{N n \times N n}$ are positive definite matrices. By simple computation, we have

$$
\begin{aligned}
& \mathcal{M}^{T} \mathbf{P} \mathcal{M}=\left[\begin{array}{cc}
M_{0}^{T} P_{11} M_{0}+\left(\tilde{M}_{0}-\bar{K} \tilde{M}\right)^{T} \mathbf{P}_{22}\left(\tilde{M}_{0}-\bar{K} \tilde{M}\right) & 0 \\
0 & 0
\end{array}\right], \\
& \mathbf{P} \mathcal{A}=\left[\begin{array}{ccc}
P_{11} A & 0 & \\
0 & \mathbf{P}_{22}\left(I_{N} \otimes A\right)-\mathbf{P}_{22} \bar{K} \tilde{C}
\end{array}\right], \\
& \mathbf{P B}=\left[\begin{array}{ccc}
P_{11} B & 0 & 0 \\
0 & \mathbf{P}_{22}\left(I_{N} \otimes B\right) & -\mathbf{P}_{22} \bar{K} \tilde{W}
\end{array}\right] .
\end{aligned}
$$

Again, by observing the terms of matrix $\Xi$ in (17) and supposing

$$
\mathbf{T}_{4}=\left[\begin{array}{ll}
T_{41} & T_{42} \\
T_{43} & \mathbf{T}_{44}
\end{array}\right]
$$

where $T_{41} \in \mathbb{R}^{n \times n}$ and $\mathbf{T}_{44} \in \mathbb{R}^{N n \times N n}$, we have

$$
\mathbf{T}_{4} \mathcal{A}=\left[\begin{array}{ll}
T_{41} A & T_{42}\left(I_{N} \otimes A-\bar{K} \tilde{C}\right) \\
T_{43} A & \mathbf{T}_{44}\left(I_{N} \otimes A-\bar{K} \tilde{C}\right)
\end{array}\right] .
$$

Obviously, to solve the problem under study more easily, one just need to take $T_{42}=0$ and $\mathbf{T}_{44}=\mathbf{P}_{22}$, which leads to the fact that

$$
\begin{aligned}
\mathbf{T}_{4} \mathcal{A} & =\left[\begin{array}{lc}
T_{41} A & 0 \\
T_{43} A & \mathbf{P}_{22}\left(I_{N} \otimes A-\bar{K} \tilde{C}\right)
\end{array}\right], \\
\mathbf{T}_{4} \mathcal{B} & =\left[\begin{array}{ccc}
T_{41} B & 0 & 0 \\
T_{43} B & \mathbf{P}_{22}\left(I_{N} \otimes B\right) & -\mathbf{P}_{22} \bar{K} \tilde{W}
\end{array}\right] .
\end{aligned}
$$

From (44)-(46) and the expression of $\Xi$ in (17), it can be seen that one needs to take $X=\mathbf{P}_{22} \bar{K}$ to make the condition in (17) convex. Based on Lemma 3 and the analysis above, with the constraint $X \in \mathscr{W}_{n \times m}$ for matrix $X$, one can easily obtain the result of this theorem. The proof is completed.

\section{REFERENCES}

Bekmezci, I. And Alagöz, F. 2009. Energy efficient, delay sensitive, fault tolerant wireless sensor network for military monitoring. Int. Journal of Distributed Sensor Networks 5, 6, 729-747.

Boyd, S., Ghaoui, L. E., Feron, E., And Balakrishnan, V. 1994. Linear matrix inequalities in system and control theory. SIAM, Philadelphia.

Brooks, R., Ramanathan, P., and Sayeed, A. 2003. Distributed target classification and tracking in sensor networks. Proceedings of the IEEE 91, 8 (Aug.), 1163-1171.

CAO, J., Chen, G., AND LI, P. 2008. Global synchronization in an array of delayed neural networks with hybrid coupling. IEEE Trans. Systems, Man and Cybernetics-Part B: Cybernetics 38, 2 (Apr.), 488-498.

Cattivelli, F. S., Lopes, C. G., And Sayed, A. H. 2008. Diffusion strategies for distributed kalman filtering: Formulation and performance analysis. In 2008 IAPR Workshop on Cognitive Information Processing. Santorini, Greece, 36-41.

Cattivelli, F. S. And Sayed, A. H. 2008. Diffusion mechanisms for fixed-point distributed kalman smoothing. In EUSIPCO. Lausanne, Switzerland, 1-4.

Cattivelli, F. S. And SAyed, A. H. 2009. Diffusion distributed kalman filtering with adaptive weights. In Asilomar Conference on Signals, Systems and Computers. Pacific Grove, CA, 908-9123.

ACM Transactions on Sensor Networks, Vol. X, No. X, XX 20XX. 
Cattivelli, F. S. And SAyed, A. H. 2010a. Diffusion LMS strategies for distributed estimation. IEEE Trans. Signal Processing 58, 3 (Mar.), 1035-1048.

Cattivelli, F. S. and Sayed, A. H. 2010b. Diffusion strategies for distributed Kalman filtering and smoothing. IEEE Trans. Automatic Control 55, 9 (Sept.), 2069-2084.

Cohen, R. And Kapchits, B. 2009. An optimal wake-up scheduling algorithm for minimizing energy consumption while limiting maximum delay in a mesh sensor network. IEEE-ACM Trans. Networking 17, 2 (Apr.), 570-581.

Egea-Lopez, E., Vales-Alonso, J., Martinez-Sala, A., Pavon-Marino, P., and GarciaHARO, J. 2006. Simulation scalability issues in wireless sensor networks. IEEE Communications Magazine 44, 7 (Jul.), 64-73.

GaO, H. And Chen, T. 2007. $H_{\infty}$ estimation for uncertain systems with limited communication capacity. IEEE Trans. Automat. Control 52, 11 (Nov.), 2070-2084.

GaO, H., Wu, J., AND Shi, P. 2009. Robust sampled-data $H_{\infty}$ control with stochastic sampling. Automatica 45, 7 (Jul.), 1729-1736.

Goodall, D. P. And Postoyan, R. 2010. Output feedback stabilisation for uncertain nonlinear time-delay systems subject to input constraints. Int. J. Control 83, 4 (Apr.), 676-693.

Karimi, H. R. And GaO, H. 2010. New delay-dependent exponential $H_{\infty}$ synchronization for uncertain neural networks with mixed time delays. IEEE Trans. Systems, Man and CyberneticsPart B: Cybernetics 40, 1 (Feb.), 173-185.

Kim, J. H., West, M., Scholte, E., and Narayanan, S. 2008. Multiscale consensus for decentralized estimation and its application to building systems. In Proceedings of the American Control Conference. IEEE, Seattle, 888-893.

Lin, C., WANG, Z., AND YANG, F. 2009. Observer-based networked control for continuous-time systems with random sensor delays. Automatica 45, 2 (Feb.), 578-584.

Liu, H., Sun, F., He, K., And Sun, Z. 2004. Design of reduced-order $H_{\infty}$ filter for Markovian jumping systems with time delay. IEEE Trans. Circuits and Systems - II: Express Briefs 51, 11 (Nov.), 607-612.

LiU, Y., WANG, Z., Liang, J., AND LiU, X. 2008. Synchronization and state estimation for discretetime complex networks with distributed delays. IEEE Trans. Systems, Man and CyberneticsPart B: Cybernetics 38, 5 (Oct.), 1314-1325.

Lu, J. Q. AND Ho, D. W. C. 2010. Globally exponential synchronization and synchronizability for general dynamical networks. IEEE Trans. Systems, Man and Cybernetics-Part B: Cybernetics 40, 2 (Apr.), 350-361.

Lu, J. Q., Ho, D. W. C., AND Wu, L. 2009. Exponential stabilization of switched stochastic dynamical networks . Nonlinearity 22, 4 (Apr.), 889-911.

MAO, X. 1997. Stochastic differential equations and their applications. Horwood, Chichester.

MaO, X., Koroleva, N., And Rodkina, A. 1998. Robust stability of uncertain stochastic differential delay equations. Syst. Control Lett. 35, 5 (Dec.), 325-336.

Meng, X., Lam, J., And GaO, H. 2009. Network-based $H_{\infty}$ control for stochastic systems . Int. J. Robust and Nonlinear Control 19, 3 (Feb.), 295-312.

Mou, S., Gao, H., Qiang, W., And Chen, K. 2008. New delay-dependent exponential stability for neural networks with time delay. IEEE Trans. Systems, Man and Cybernetics-Part B: Cybernetics 38, 2 (Apr.), 571-576.

Mou, S., GaO, H., ZhaO, Y., And QIANG, W. 2008. Further improvement on synchronization stability of complex networks with coupling delays. Int. J. Comput. Math. 85, 8 (Aug.), 12551263.

Naghshtabrizi, P. and Hespanha, J. P. 2005. Designing an observer-based controller for a network control system. In 2005 44th IEEE Conference on Decision and Control and European Control Conference. IEEE, Spain, 848-853.

Olfati-Saber, R. 2007. Distributed kalman filtering for sensor networks. In Proceedings of the 46th IEEE Conference on Decision and Control. IEEE, New Orleans, 1814-1820.

Olfati-Saber, R. and Shamma, J. S. 2005. Consensus filters for sensor networks and distributed sensor fusion. In 2005 44th IEEE Conference on Decision and Control and European Control Conference. IEEE, Spain, 6698-6703. 
Schuss, Z. 1980. Theory and applications of stochastic differential equations. Wiley, New York. Shen, B., Wang, Z., Hung, Y. S., And Chesi, G. 2011. Distributed $H_{\infty}$ filtering for polynomial nonlinear stochastic systems in sensor networks. IEEE Transactions on Industrial Electronics 58, 5 (May), 1971-1979.

Shi, P., Mahmoud, M., NGuang, S., and Ismail, A. 2006. Robust filtering for jumping systems with mode-dependent delays. Signal Processing 86, 1 (Jan.), 140-152.

Sinopoli, B., Sharp, C., Schenato, L., Schaffert, S., and Sastry, S. S. 2003. Distributed control applications within sensor networks. Proceedings of the IEEE 91, 8 (Aug.), 1235-1246.

Song, Q. AND CAO, J. 2007. Impulsive effects on stability of fuzzy Cohen-Grossberg neural networks with time-varying delays. IEEE Trans. Systems, Man and Cybernetics-Part B: Cybernetics 37, 3 (Jun.), 733-741.

Speranzon, A., Fischione, C., Johansson, K. H., and Sangiovanni-Vincentelli, A. 2008. A distributed minimum variance estimator for sensor networks. IEEE Journal on Selected Areas in Communications 26, 4 (May), 609-621.

WANG, X., WANG, S., AND BI, D. 2009. Distributed visual-target-surveillance system in wireless sensor networks. IEEE Trans. Systems, Man and Cybernetics-Part B: Cybernetics 39, 5 (Oct.), $1134-1146$

WANG, Y., WANG, Z., AND LIANG, J. 2008. A delay fractioning approach to global synchronization of delayed complex networks with stochastic disturbances. Phys. Lett. A 372, 39 (Sep.), 60666073.

WANG, Y., WANG, Z., AND LIANG, J. 2009. Global synchronization for delayed complex networks with randomly occurring nonlinearities and multiple stochastic disturbances. J. Phys. A: Math. Theor. 42, 13 (Apr.).

WANG, Z., WANG, Y., AND LiU, Y. 2010. Global synchronization for discrete-time stochastic complex networks with randomly occurred nonlinearities and mixed time delays. IEEE Trans. Neural Networks 21, 1 (Jan.), 11-25.

Wu, L., Shi, P., GaO, H., And Wang, C. 2008. $H_{\infty}$ filtering for 2D Markovian jump systems. Automatica 44, 7 (Jul.), 1849-1858.

Xiong, J. And Lam, J. 2006. Fixed-order robust $H_{\infty}$ filter design for Markovian jump systems with uncertain switching probabilities. IEEE Trans. Signal Processing 54, 4 (Apr.), 1421-1430.

Xiong, Z., Liveris, A. D., And Cheng, S. 2004. Distributed source coding for sensor networks. IEEE Signal Processing Magazine 21, 5 (Sep.), 80-94.

Yu, W., Chen, G., Wang, Z., And Yang, W. 2009. Distributed consensus filtering in sensor networks. IEEE Trans. Systems, Man and Cybernetics-Part B: Cybernetics 39, 6 (Dec.), $1568-1577$.

YuAN, K., CAO, J., AND LI, H. X. 2006. Robust stability of switched Cohen-Grossberg neural networks with mixed time-varying delays. IEEE Trans. Systems, Man and Cybernetics-Part B: Cybernetics 36, 6 (Dec.), 1356-1363.

Yue, D., Han, Q., AND Peng, C. 2004. State feedback controller design of networked control systems. IEEE Transactions on Circuits and Systems II: Express Briefs 51, 11 (Nov.), 640-644.

Yue, D., Tian, E., Wang, Z., And Lam, J. 2009. Stabilization of systems with probabilistic interval input delays and its applications to networked control systems. IEEE Trans. Systems, Man and Cybernetics-Part A: Systems and Humans 39, 4 (Jul.), 939-945.

ZeČević, A. I. AND ŠILJAK, D. D. 2004. Design of robust static output feedback for large-scale systems. IEEE Trans. Automat. Control 49, 11 (Nov.), 2040-2044.

Zhang, H., Feng, G., Duan, G., and Lu, X. 2006. $H_{\infty}$ filtering for multiple-time-delay measurements. IEEE Trans. Signal Processing 54, 5 (May), 1681-1688.

Received September 2010; revised XX 20XX; accepted XX 20XX 